
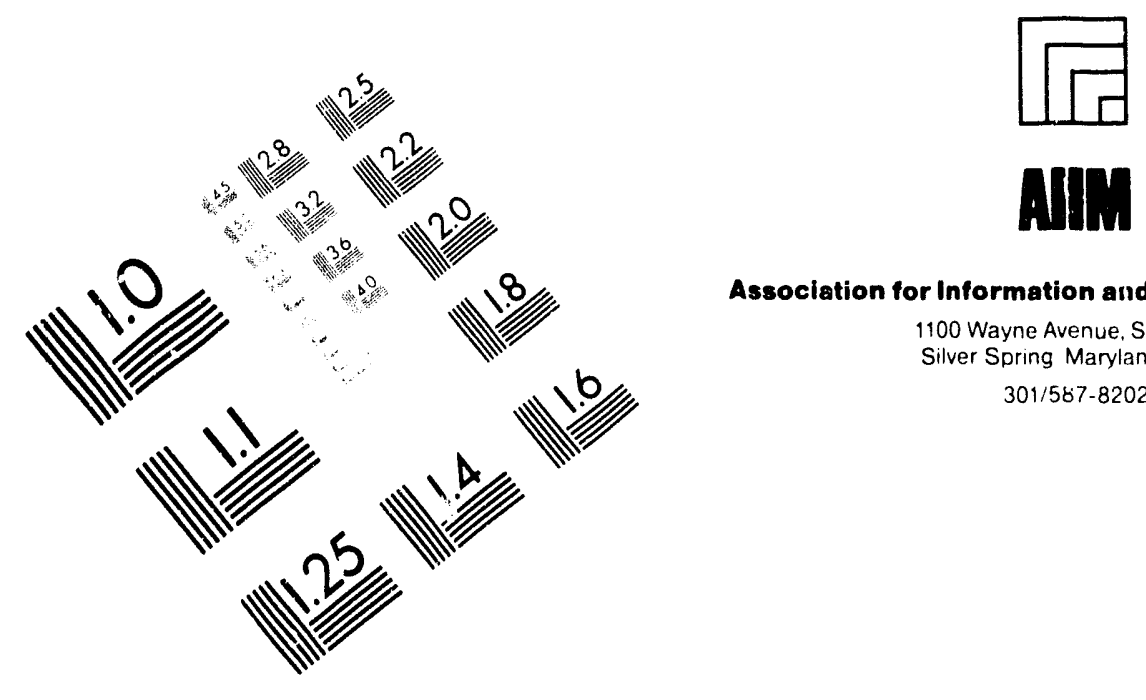

Association for Information and Image Management

1100 Wayne Avenue. Suite 1100

Silver Spring Maryland 20910

301/587-8202

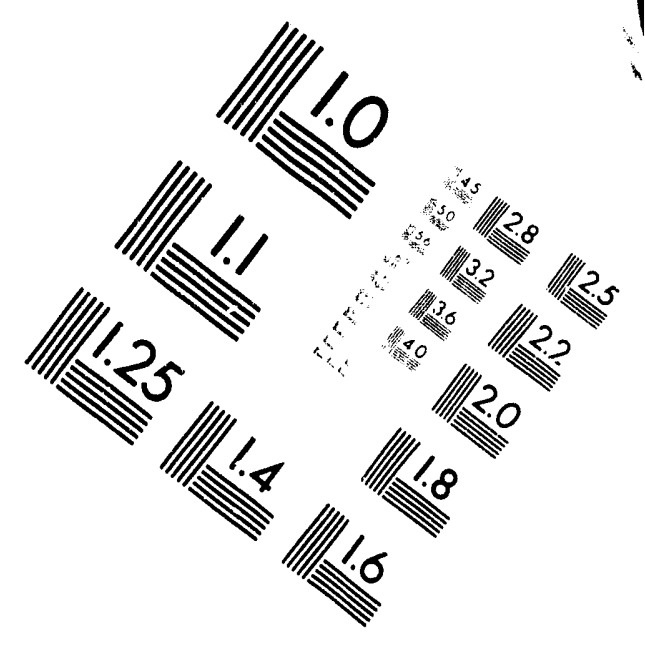

\title{
Centimeter
}

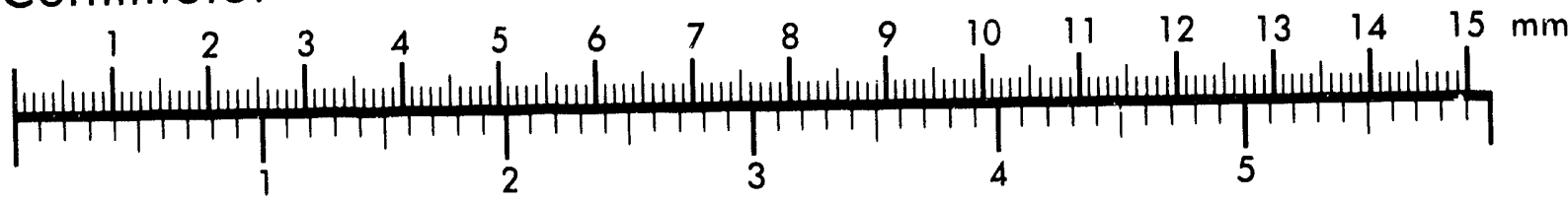
Inches
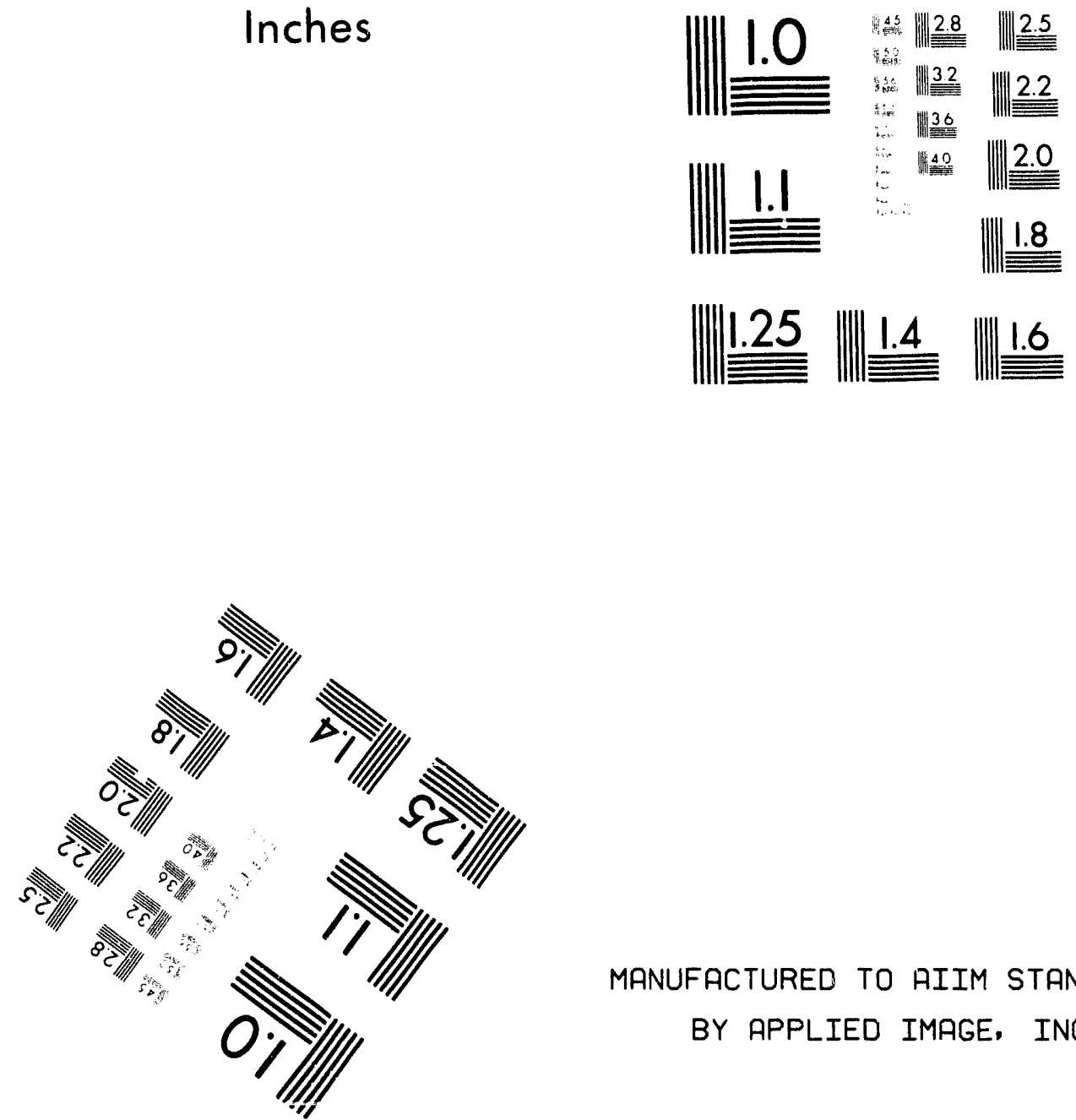

MANUFACTURED TO AIIM STANDARDS

BY APPLIED IMAGE, INC.

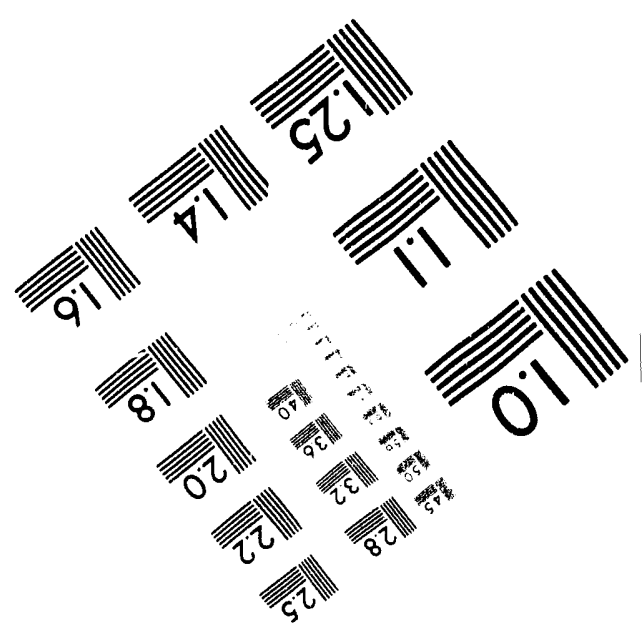



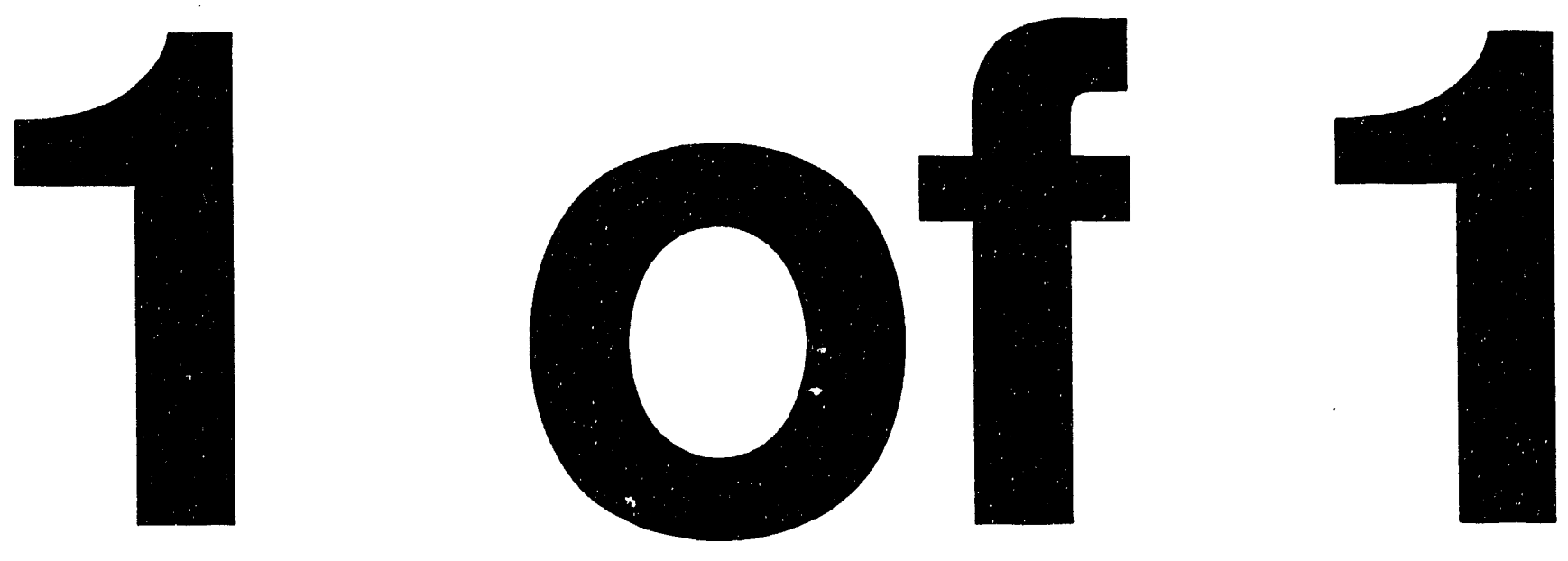
LBL-33257

CBP-225

UC-414

\section{The Standing Wave FEL/TBA: Realistic Cavity Geometry and Energy Extraction*}

Jin-Soo Kim, Heino Henke, Andrew M. Sessler and William M. Sharp

Lawrence Berkeley Laboratory, University of California, Berkeley, California 94720

May 1993

* Work supported by the Director, Office of Energy Research, Office of High Energy and Nuclear Physics, Division of High Energy Physics, of the U.S. Department of Energy under Contract No. DE-AC0376SF00098 


\title{
The Standing Wave FEL/TBA: Realistic Cavity Geometry and Energy Extraction*
}

\author{
Jin-Soo Kim, Heino Henke (a), Andrew M. Sessler, and William M. Sharp ${ }^{(b)}$ \\ Lawrence Berkeley Laboratory \\ University of California, Berkeley, California 94720
}

Abstract

A set of parameters for standing wave free electron laser two beam accelerators (SWFEL/TBA) is evaluated for realistic cavity geometry taking into account beam-break-up and the sensitivity of output power to imperfections. Also given is a piwer extraction system using cavity coupled wave guides.

\section{INTRODUCTION}

For the next generation linear colliders, a high gradient acceleration structure is necessary. As a possible source of energy for such colliders, a SWFEL/TBA has been proposed[1,2]. A schematic diagram of a FEL/TBA is shown in Figure 1.

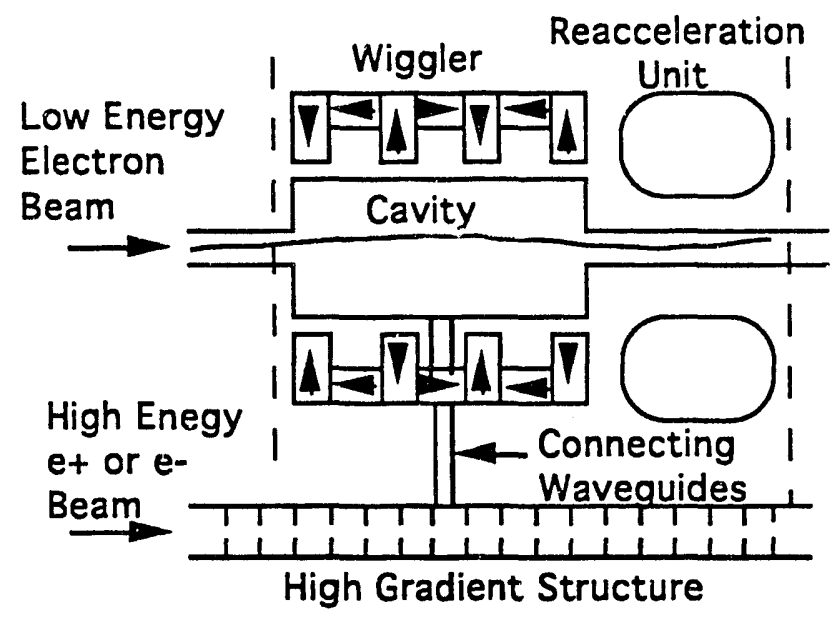

Figure 1. Schematic diagram of SWFEL/TBA

There have been some parameter studies of SWFEL/TBA parameters. Preliminary optimizations were made for the constancy of output energy and phase with respect to de-tuned energy for short pulse bunches[3]. The sensitivity has been reduced further by utilizing drift tubes between the cavities[4].

In this paper, we optimize the parameters with

\footnotetext{
* The work was supported by the Director, Office of Energy Research, Office of High Energy and Nuclear Physics, Division of High Energy Physics, of the U. S. Department of Energy under Contract No. DE-AC03-76SF00098.

(a) Permanent address: Technische Universitat Berlin, Institute fur Theoretische Electrotechnik, Einsteinufer 17, D-1000 Berlin 10, Germany.

(b) Permanent address: Lawrence Livermore National Laboratory, University of California, Livermore, Califormia 94550, USA.
}

longer pulses (for better efficiency), with longer cavities (for BBU considerations [5]) than previously considered, and with realistic engineering constraints. Induction linacs have leakage current the order of $100 \mathrm{~A}$ and need much higher current than this level to be efficient. Switching time for such a high current takes about $10 \mathrm{nsec}$ and thus beam pulse lengths significantly longer than this are needed for a good efficiency. An output energy of about $10 \mathrm{~J} / \mathrm{m}$ is desired. Reacceleration can also not be too large, since about $30 \mathrm{~cm}$ long ferrite material is needed for $0.25 \mathrm{MeV}$ reacceleration. With these constraints transverse and longitudinal beam dynamics are considered and lead to a set of parameters for SWFEL/TBA.

\section{B.ASIC MODEL}

Within a cavity the particles and fields of a SWFEL can be examined by the conventional wiggle-averaged FEL equations[6]. Using the subscripts $s$ for signal waves and $w$ for wiggler quantities, and representing the vector potential by the usual normalized quantity $a=A /\left(m c^{2} / e\right)$, the equations for particle phase, $\theta_{j}$, the $j$-th particle energy, $\gamma_{j}$, and the field amplitude, $a_{S}$, with phase, $\phi$, are given by the following.

$$
\begin{aligned}
& \frac{d \vartheta_{j}}{d z}=k_{s}+k_{w}-\frac{\omega_{s}}{c}-\frac{\omega_{s}}{2 c \gamma_{j}^{2}}\left[1+\frac{a_{w}^{2}}{2}-2 D_{x} a_{w} a_{s} \cos \left(\vartheta_{j}+\phi\right)\right] \\
& \frac{d \gamma_{j}}{d z}=-D_{x} \frac{\omega_{s}}{c} \frac{a_{w}}{\gamma_{j}} a_{s} \sin \left(\vartheta_{j}+\phi\right) \\
& \frac{d\left(a_{s} e^{i \phi}\right)}{d s}=i c\left(\frac{2 \gamma_{r}^{2}}{a_{w}}\right)\left(\frac{R}{Q}\right)\left(\frac{I}{I_{a}}\right)\left(\frac{e^{-i \vartheta_{j}}}{\gamma_{j}}\right)
\end{aligned}
$$

Here, $\gamma_{r}$ is the resonant beam energy, $I$ is the average beam current, $z$ is the axial coordinate, $s$ is the distance from the leading bunch, and the jitter term $D_{X}=1 / 2$. The angle brackets are averages over particles of a bunch. The brackets are redundant since in our analysis we make the approximation that only one macro particle per bunch. The shunt impedance is then given by

$$
\left.\left.\frac{R}{Q} \equiv \frac{4 \pi}{V L \omega}\right|_{-L / 2} ^{+L / 2} d z \frac{\bar{v}(z)}{v_{z}} \cdot \bar{a}(z) \exp \left(-\frac{i \omega z}{v_{z}}\right)\right|^{2},
$$

where $\bar{v}$ is the particle velocity, $v_{z}$ is the $z$ component of the velocity, $\omega$ is the FEL mode angular frequency, $V$ is the volume of the cavity, and $L$ is the length of the cavity.

Once a particle leaves a cavity, the FEL field bounces back to the other end of the cavity as a standing wave. Thus, the field should be updated accordingly. 
The bunches in a pulse are then considered as blocks of $2 \mathrm{~N}$. Since the particles do not interact much with the reflected waves, the first $2 \mathrm{~N}$ bunches behave identically where $\mathrm{N}=\mathrm{L} / \mathrm{c}$. The next $2 \mathrm{~N}$ bunches see the reflected field of the previous $2 \mathrm{~N}$ bunches. Thus we need to examine only one bunch per every $2 \mathrm{~N}$ bunches. Utilizing the drift tubes between cavities, and by adjusting the beam energy for each $2 \mathrm{~N}$ particles, it is possible to make each $2 \mathrm{~N}$ particles behave identically through each cavity, thus yielding a stable high power energy for a long device as described in Reference [4].

The output energy is then proportional to the square of $R / Q$ and the total charge of the pulse $I L_{p}$.

$W_{\text {out }} \propto\left(I L_{p}\right)^{2}\left(\frac{R}{Q}\right)^{2}$.

The proportionality consk .t not shown in the above equation, depends on the geometry of the cavity.

\section{GEOMETRY EFFECT}

The geometry of cavities and irises affect the field equation, the third equation of Eq.(1), through $R / Q$. Although the quantity $R / Q$ depends on the beam energy and wiggler fields, the geometry dependence affects the FEL performance through this quantity only. Thus, we can evaluate FEL performance for various geometry by simply evaluating this quantity and using the usual FEL equations.

Now it is easy to understand the geometry effect since the geometry term affects only the field equation, and at the same time the averaged beam current affects only the field equation. Therefore, the geometry effect can be easily compensated by adjusting current, keeping $I R / Q$ constant. Thus sensitivity of output power to various errors is as in an ideal cavity. The output energy may be different. This, however, is not a problem since it is necessary to have constant output energy but the magnitude of the energy is not so important.

\section{NUMERICAL RESULTS}

Eq.(1) is advanced by the fourth order Runge-Katta method. Assuming no slippage between particles and fields, the field equations are evolved in $z$ (replacing $s$ by $z$ ). The initial field phase was set to zero and the initial particle phase is given as $\pi / 3$.

A typical evolution of FEL variables, in the absence of errors, are illustrated in Figure 2. In the absence of any errors, the dynamics of each $2 \mathrm{~N}$ block is the same for the cavities. Thus only the first 3 cavities are shown. The particle lose energy (a) as it traverses a cavity while the field amplitude increases (b). The lost energy of the particle is replenished before entering the next cavity, thus repeating the same motion again. In (c) particle phase, field phase, and the ponderomotive phase are shown.

For stable FEL performance, it is important to keep the pondromotive phase $\psi=\theta_{j}+\phi$ invariant. In principle we do not have to consider phase change of $\theta$ and $\phi$ independently. However, best results are obtained by minimizing the variation of the particle phase within a cavity, by making the nonsinusoidal component of the particle phase equation in Eq.(1) small. For $17.1 \mathrm{GHz}, k_{w}=0.16$ is good.

A set of parameters is summarized in Table 1, taking into account BBU, beam sensitivity and engineering

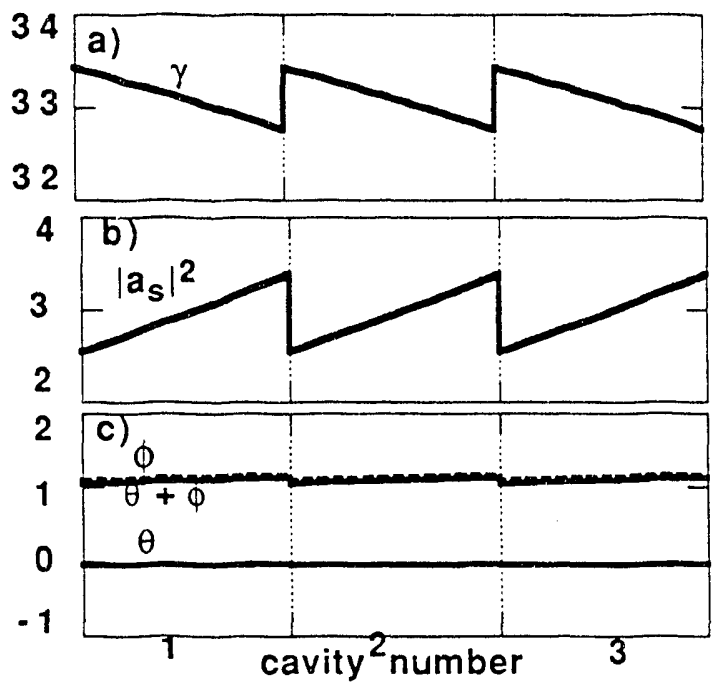

Figure 2. Particle energy (a), field energy (b), particle phase, field phase, and the ponderomotive phase (c) of $\mathrm{j}$-th particle for the first three cavities. Drift tube regions are not shown, and simply indicated as a vertical dotted line.

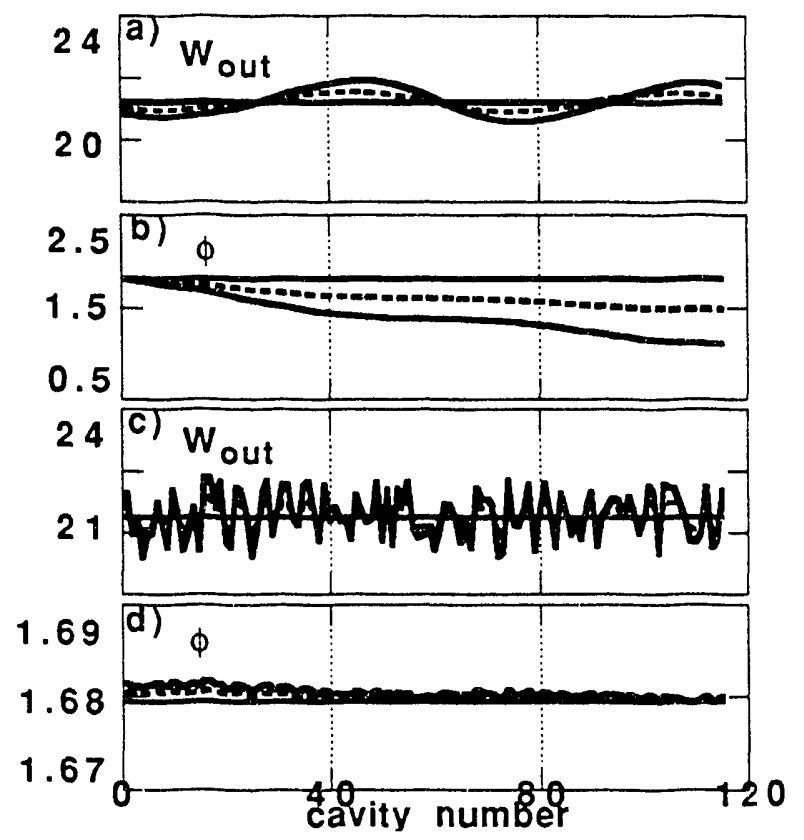

Figure 3. Output energy (a) and field phase (b) versus device length for $0 \%$ (straight line), $0.5 \%$ (dotted curve), and $1 \%$ (solid curve) de-tune in beam energy for the parameters in Table 1. The same quantities are shown in (c) and (d) for $0 \%$ (straight line), $2 \%$ (dotted curve), and $4 \%$ (solid curve) for random cavity errors in $R / Q$. 
constraints. For the parameters shown in Table 1, sensitivity of output energy and field phase to energy de-tune and local field errors are shown in Figure 3. It shows 6\% fluctuation in output energy (a) and 0.7 radian phase fluctuation (b) with $1 \%$ energy de-tune. With $0.5 \%$ energy de-tune, the fluctuations are half of those $1 \%$ de-tune case. Similar results are obtained with half the cavity length but twice as many cavities. Imperfection in cavities are introduced as field errors in each cavity. When the input power was increased to $80 \mathrm{~kW} / \mathrm{m}$, the output energy fluctuation remained the same and the phase fluctuation was reduced to half. With $4 \%$ random variations of the field errors we obtained $4 \%$ energy fluctuation (c) and 0.0025 radian phase fluctuations (d). The reason for the small phase fluctuations is that the particle phase was adjusted with local field errors. With $2 \%$ field errors, the fluctuations are reduced by haif.

Table 1. Parameters of a SWFEL for $17.1 \mathrm{GHz}$

\begin{tabular}{ll}
\hline wiggler & parameter $a_{w}=1.41$ \\
& $\begin{array}{l}\text { B field }=0.9 \mathrm{kG} \\
\text { wiggler period } \lambda_{\mathrm{w}}=39 \mathrm{~cm} ;\left(k_{w}=0.1598\right) \\
\text { device length }=100 \mathrm{~m}\end{array}$ \\
\hline cavity length & $86 \mathrm{~cm}$ \\
\hline beam & $\begin{array}{l}\text { energy } \gamma=33.1 \\
\text { average current } I=1 \mathrm{kA} \\
\text { pulse length }=100 \mathrm{~ns}\end{array}$ \\
\hline input power & $8 \mathrm{~kW} / \mathrm{m}$ \\
\hline output energy & $21.2 \mathrm{~J} / \mathrm{m}$ \\
\hline
\end{tabular}

\section{RF POWER EXTRACTION}

The RF energy is extracted from both sides of the rectangular cavity, to rectangular wave guides coupled by circular holes. Assuming that the operating mode of the SWFEL is TE $01 \mathrm{p}$, then the wave guide modes are excited by $\mathrm{TE}_{10 \mathrm{p}}$ mode, and propagate to both ends. Let $a$ be the width, $b$ is the height, $L$ is the length of the cavity, then $b$ is the width of the guide. (The height of the cavity shares the width of the wave guides.)

$$
\begin{aligned}
& \bar{E}_{01 p}=E_{0} \sin \left(\frac{\pi y}{b}\right) \sin \left(\frac{p \pi z}{L}\right) \hat{x} ; \text { in cavity, } \\
& \bar{E}_{10 p}^{ \pm}=D_{0} \sin \left(\frac{\pi y}{b}\right) e^{ \pm i k_{z} z} \hat{x} ; \text { in waveguides, }
\end{aligned}
$$

where $E_{0}$ and $D_{0}$ are amplitude of the modes and $k_{z}=p \pi / L$. The holes are located at the maximum magnitude of the operating mode. If the coupling hole radius $r$ is small compared to the $z$-wavelength of the cavity mode, each hole behaves like an electric dipole source to the wave guide. Then following Reference 7, we can estimate the output power per port as follows.

$$
P_{o u t}^{ \pm}=\frac{1}{2}\left|-\frac{i \omega}{2} \vec{E}_{10}^{ \pm} n \vec{P}\right|^{2} ; \quad \vec{P}=-\frac{2}{3} \varepsilon_{o} r^{3} E_{o} e^{-\alpha t} \hat{x} .(5)
$$

The exponent $\alpha$ is the attenuation of the dipole due to the thickness $t$ of the cavity / wave-guide wall, given by $\alpha=\left(\left(J_{o 1} / r\right)^{2}-k^{2}\right)^{1 / 2}$, with $k$ being the wave-number of the operating FEL mode in free space. It is assumed that all the $n$-holes are in phass.

Noting that the amplitude of the wave guide mode $D_{0}=\sqrt{Z_{1}} \sqrt{2 / b c}$ with $Z_{1}=k_{z} /\left(\omega \varepsilon_{0}\right)$ we can determine the radius of holes to extract the energy in the cavity within $\tau=100$ nsec. Since the energy in a cavity is $U=\varepsilon_{O}(a b L / 8) E_{O}^{2}$, and $P_{o u t}=U / 4 \tau$ where the factor 4 in the denominator came from 4 ports, we obtain

$$
P_{o u s}^{ \pm}=\frac{n^{2} k^{2} r^{6}}{9 b c Z_{0}}\left(1-\left(\frac{\pi}{k b}\right)^{2}\right)^{1 / 2} e^{-2 \alpha t} E_{0}^{2}=\frac{\varepsilon_{0} a b L}{32 \tau} E_{0}^{2} .
$$

The above equation gives a relation between geometry of the cavity and the extraction time. Note that the output power is proportional to 6 , apart from an $r$ dependence in $\alpha$.

For $17.1 \mathrm{GHz}, a=10 \mathrm{~m}, b=3 \mathrm{~cm}, c=1.5 \mathrm{~cm}, t=0.5$ $\mathrm{cm}$, with $n=60$ we find that the radius of the hole to be 0.47 $\mathrm{cm}$. For $11.4 \mathrm{GHz}$, if we keep all other parameters fixed, the radius is $0.37 \mathrm{~cm}$.

\section{CONCLUSION}

We propose a set of parameters for a SWFEL/TBA in Table 1. The parameters are chosen taking into account engineering constraints, transverse beam displacements, and beam sensitivity. Also presented is a calculation which shows hat the cavity energy can be extracted adequately through cavity coupled wave guides.

\section{REFERENCES}

[1] A.M.Sessler, in Laser Acceleration of Particles, edited by P.J.Channell (AIP, New York,1982), p.154.

[2] A.M.Sessler, D.H.Whittum, J.S.Wurtele, W.M.Sharp, and M.A.Makowski "Standing -wave free-electron laser two-beam acceleratior, Nucl. Inst, Method in Phys. Res. A306, 592 (1991). Eq.(32) should be corrected by a factor of $1 / 16$.

[3] W.M.Sharp, G. Rangarajan, A. M. Sessler, and J.S. Wurtele, Proc. SPIE Conference 1407, p.535 (1991).

[4] G.Rangarajan and A.M.Sessler, " $\$$ risitivity studies of a standing-wave free-electron lase:", Proc. of the Third Workshop on Advanced Accelerator Concepts, Port Jefferson, June 1992 (to be published); LBL preprint, LBL-32463, (1992).

[5] J.S.Kim, A.M.Sessler and D.H.Whittum and H.Henke, "Multi-Bunch BBU(Beam-Break-Up) Studies in a SWFEL/TBA", This proceeding Pa14.

[6] N.M. Kroll, P.L. Morton, and M.N. Rosenbluth, "FreeElectron Lasers with variable parameter wigglers", IEEE J.Quantum Electron. QE-17 p.1436 (1981).

[7] R.E.Collins, "Foundations for microwave engeneerings", McGraw Hill, NY, (1966). 

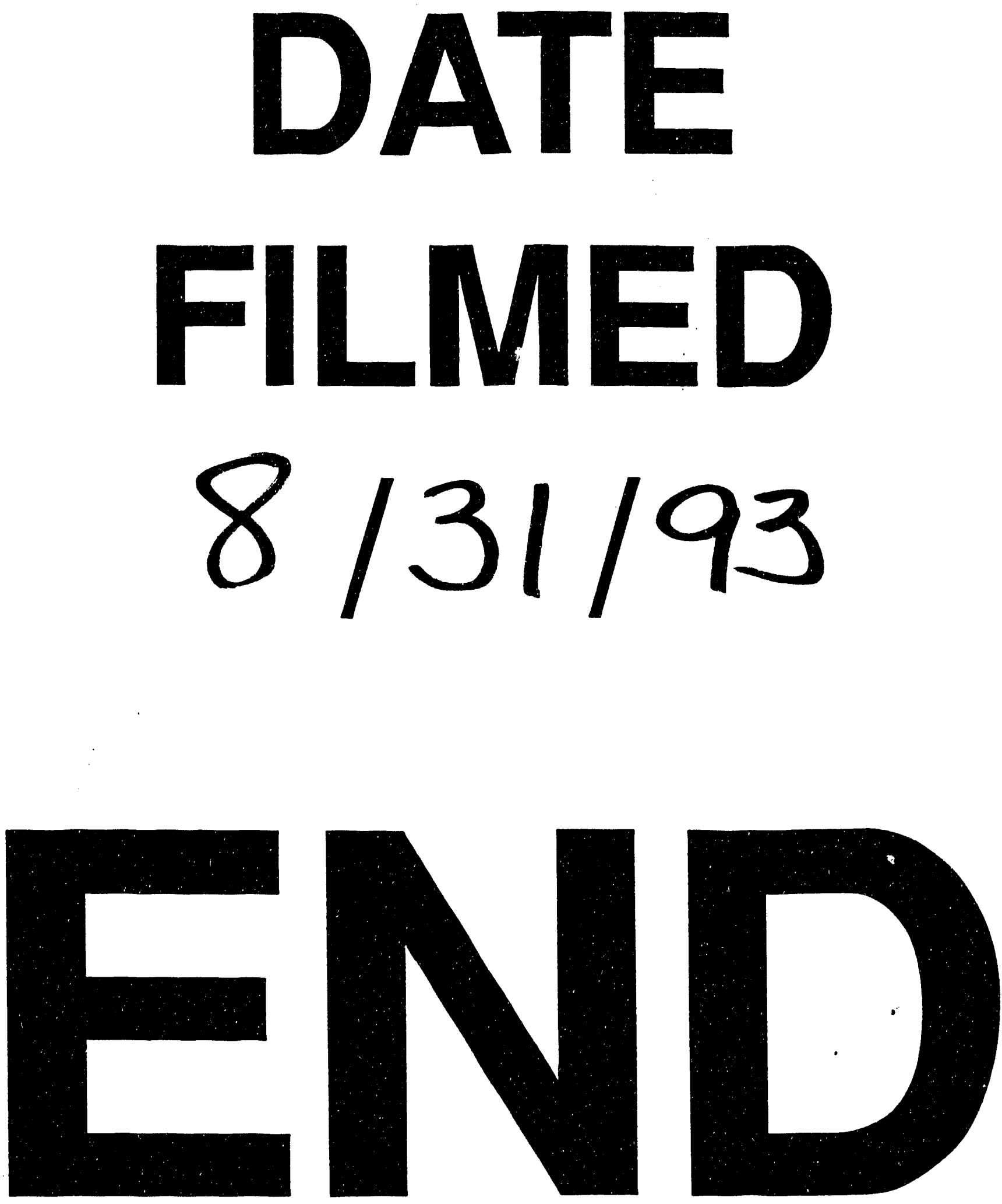
